\title{
Scapular deformity in obstetric brachial plexus palsy: a new finding
}

\author{
Rahul K. Nath • Melia Paizi
}

Received: 24 June 2006 / Accepted: 29 November 2006 / Published online: 30 January 2007

(C) Springer-Verlag 2007

\begin{abstract}
While most obstetric brachial plexus palsy patients recover arm and hand function, the residual nerve weakness leads to muscle imbalances about the shoulder which may cause bony deformities. In this paper we describe abnormalities in the developing scapula and the glenohumeral joint. We introduce a classification for the deformity which we term Scapular Hypoplasia, Elevation and Rotation. Multiple anatomic parameters were measured in bilateral $\mathrm{CT}$ images and three-dimensional CT reconstruction of the shoulder girdle of 30 obstetric brachial plexus palsy patients (age range 10 months-10.6 years). The affected scapulae were found to be hypoplastic by an average of $14 \%$ while the ratio of the height to the width of the body of scapula (excluding acromion) were not significantly changed, the acromion was significantly elongated by an average of $19 \%$. These parameters as well as subluxation of the humeral head (average 14\%) and downward rotation in the scapular plane were found to correlate with the area of scapula visible over the clavicle. This finding provides a classification tool for diagnosis and objective evaluation of the bony deformity and its severity in obstetric brachial plexus palsy patients.
\end{abstract}

Keywords Brachial plexus $\cdot$ Birth injuries $\cdot$ Scapula Acromion $\cdot$ Paralysis $\cdot$ Obstetric

R. K. Nath $(\varangle) \cdot$ M. Paizi

Texas Nerve and Paralysis Institute,

2201 W. Holcombe Blvd., Ste. 225,

Houston, TX 77030, USA

e-mail:drnath@drnathmedical.com

\section{Introduction}

The most commonly injured brachial plexus nerve root in obstetric injuries is the fifth cervical (C5) root. The supplied shoulder girdle musculature is therefore most commonly affected and indeed shoulder deformities comprise the most significant problems in this injury [2]. Within the context of shoulder deformity, the medial rotation contracture (MRC) deformity results in the greatest morbidity. The pathophysiology of deformity has been known for over 100 years: paralysis of the shoulder abductors and external rotators leads to relative dominance of already strong internal rotators of the shoulder, with eventual clinically observed changes of arm posture. The imbalance causes progressive deformity in the growing shoulder which may progress to a fixed medial rotation position of the humerus as well as posterior subluxation of the humeral head [3]. This constant position of the shoulder has a deleterious effect on glenohumeral development $[9,14,17]$ and is accompanied by a characteristic flexion and MRC of the shoulder. Furthermore, scapular growth is impaired compared to the normal side [12]. The patients exhibit a persistent elbow-bent posture, pronation position of the forearm, and apparent shortening of the arm (Fig. 1). In movement, there is loss of supination due to the abnormally situated arm in medial rotation, obvious elbow flare when the biceps is flexed, the "bugler's position", and awkward external rotation. This secondary structural shoulder deformity develops early and may persist despite improvement in neurological status [14].

There have been previous radiologic demonstrations of scapular deformity as a sequela of obstetric brachial plexus palsy using plain radiographs or computed axial 


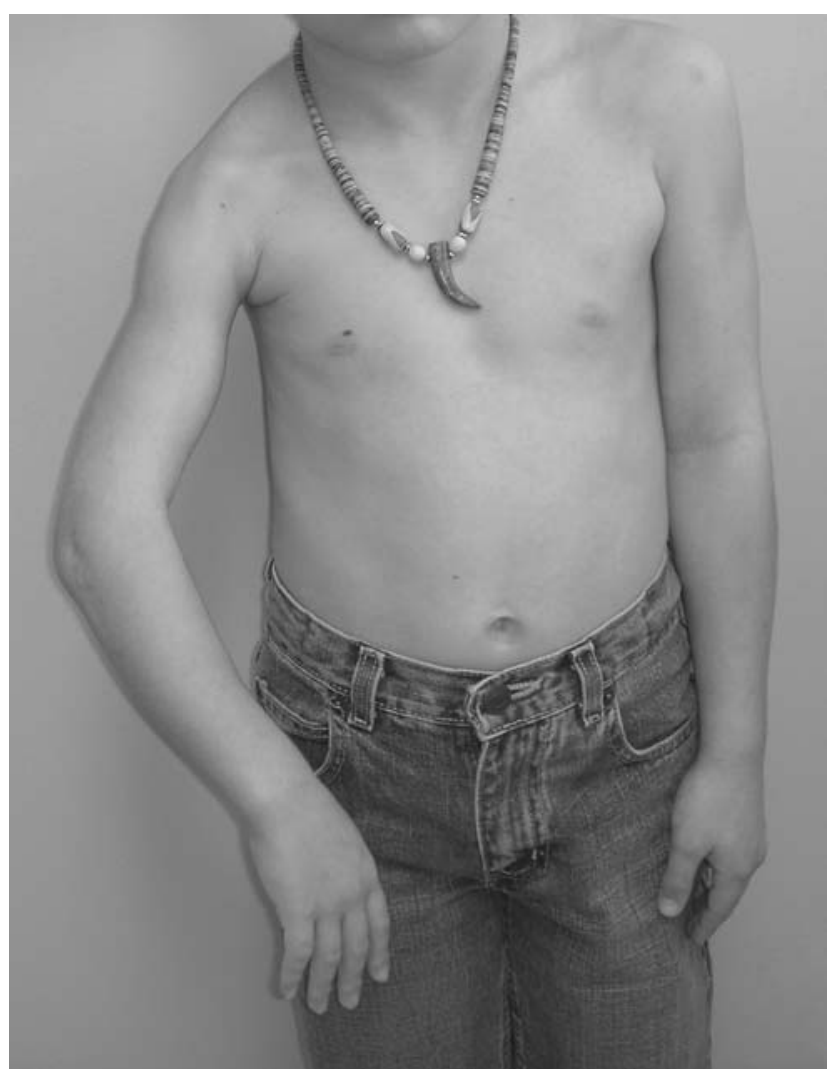

Fig. 1 At rest the child is noted to exhibit an internal rotation posture at the shoulder with elbow flexion and pronation of the forearm. There is apparent shortening of the arm

tomography were used, but attention was directed solely to the glenoid fossa and adjacent structures, without evaluating the body, spine and acromion process $[8,11,12,17]$.

In the current study, we describe dysplasia and malposition of the entire scapula in obstetric brachial plexus palsy patients. We introduce a classification of this deformity which we term collectively Scapular Hypoplasia, Elevation and Rotation (SHEAR).

\section{Methods}

\section{Patients}

A total of 30 obstetric brachial plexus palsy patients with glenohumeral internal rotation were evaluated in the past 6 months. We reviewed the clinical data on these patients and diagnosed and classified the scapular elevation according to the results of clinical examination and analysis. There were 10 boys and 20 girls ranging in age from 10 months to 10.6 years. Three of the children had not undergone any surgery. Twenty-four of the children had undergone latissimus dorsi and teres major muscle transfer, subscapularis, pectoralis major and minor contracture releases and axillary nerve decompression and neurolysis for correction of abduction at our institute in the past. Twelve of the latter group and one that had not undergone the aforementioned procedure had undergone primary neurological repair surgery.

\section{Measurements}

Transverse CT section and three-dimensional reconstructions of bilateral computerized tomograms (3D-CT) were used in the evaluation process. Trunk anterior and trunk posterior views of the whole shoulder girdle as well as superior trunk outlet and scapular posterior and medial views were examined. The contralateral scapulae were assessed for comparison.

The height $(H)$ of the scapula was measured on the scapular medial view as the length of the medial border between the superior angle and the inferior angle. Width was measured along the spine of the scapula on the scapular posterior view. The total width $(W)$ was measured from the lateral end of the acromion to the most medial part of the scapula. Width of the body of the scapula $(w)$ was measured as the distance from the glenoid to the most medial part of the scapula (Fig. 2a).

Vertical displacement was measured on the trunk posterior view with reference to the contralateral side. Lines were drawn from the center of the glenoid cavity perpendicular to the vertebral axis line. Vertical displacement is the percentage of the difference between the levels of the two glenoids $(A)$ divided by the height of the contralateral scapula $(H)$. Positive sign denotes superior displacement and negative sign inferior displacement (Fig. 2b).

In order to define the rotation of the affected scapula on an axis perpendicular to the scapular plane (downward/upward) we calculated the angle $(\alpha)$ between the extension of the line connecting the mid glenoid to the base of the spine of the scapula and the vertebral axis line (Fig. 2b). We also calculated two angles between three lines forming a triangle: Line 1 was drawn between the sterno-clavicular joint and the center of the acromio-clavicular joint, and line 2 between the center of the acromio-clavicular joint and the inferior angle of the scapula. Line 3 was the vertebral axis line. The superior scapular angle $(\gamma)$ is the angle defined between lines 1 and 2 and the inferior scapular angle $(\delta)$ is the angle between lines 2 and 3 (Fig. 2c). The rotation on the axis of the scapular spine (anterior/posterior) was assessed on the superior outlet view by measuring the angle $(\phi)$ of convergence of the longitudinal lines of the shaft of the 

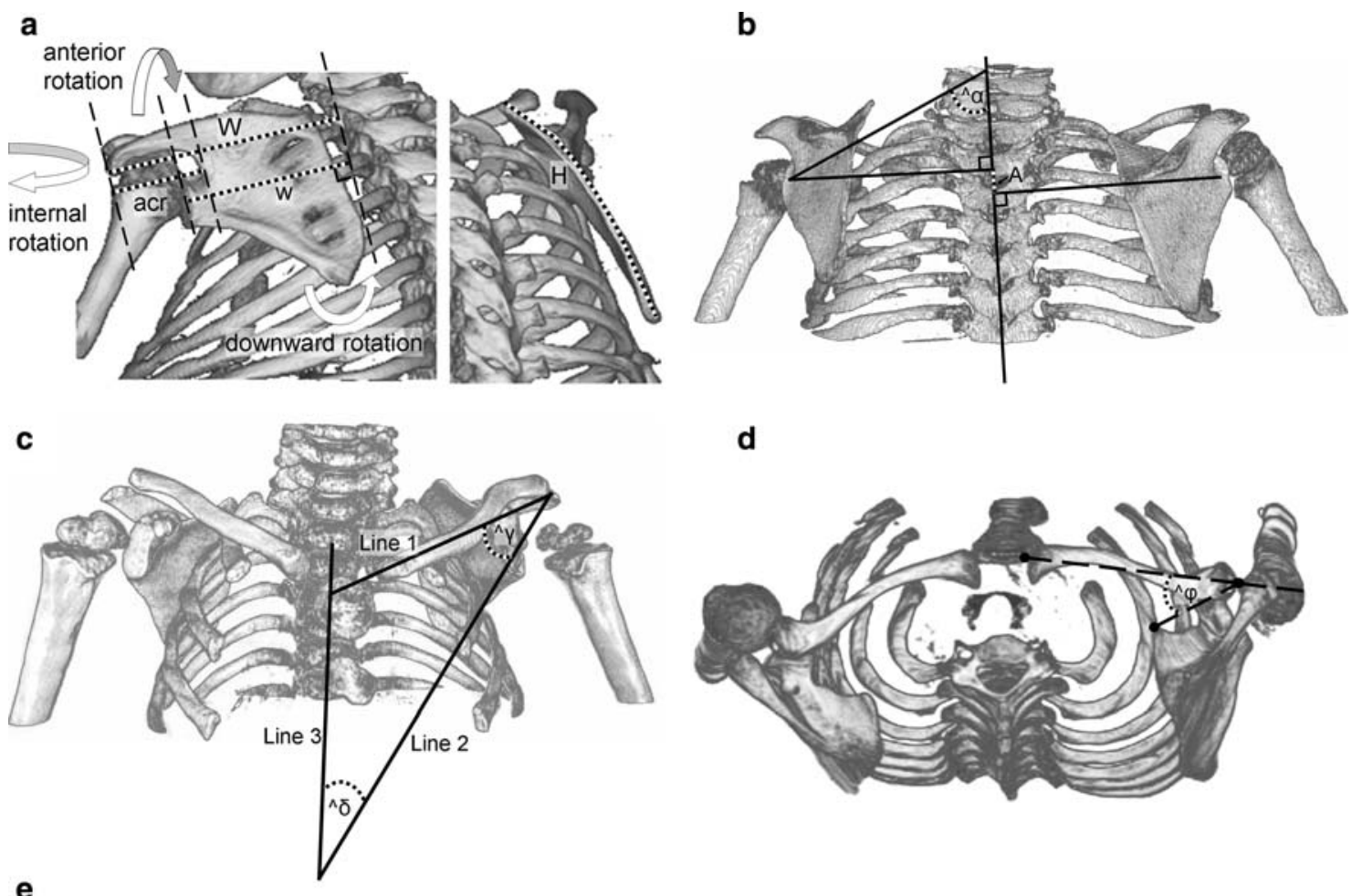

d
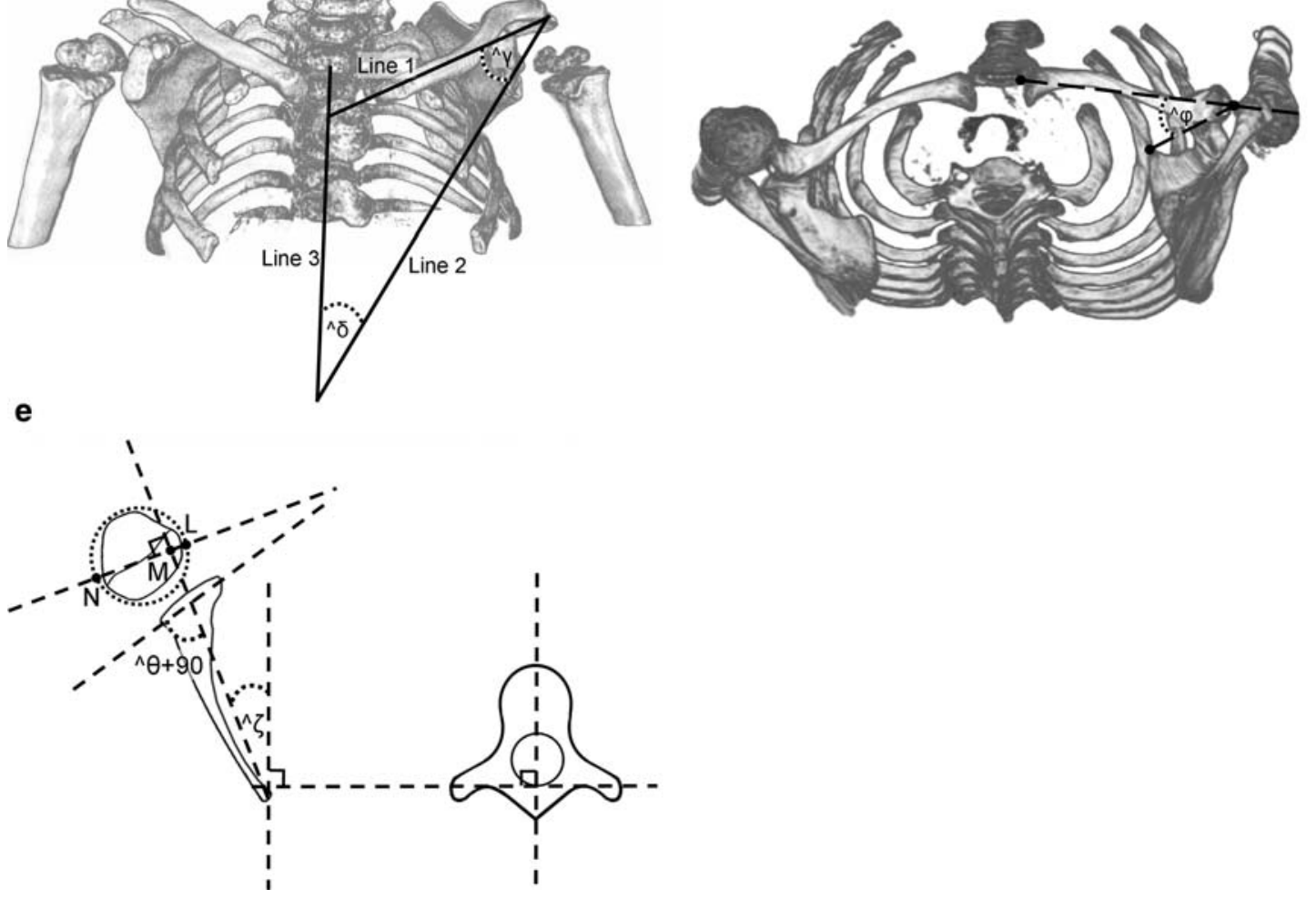

Fig. 2 Measurement of the different scapular variables. a The width is assessed in the posterior scapular view (left) along the axis of the scapular spine. The total width $(W)$ is calculated as the length from the end of the acromion to the most medial aspect of the scapula. The scapular body width $(w)$ is calculated as the distance from the mid glenoid to the most medial aspect of the scapula. The acromion length $(a c r)$ is also calculated on this view. The height $(H)$ is measured along the medial border of the scapula in the medial scapular view (right). b Lines are drawn from the center of the glenoid cavity perpendicular to the vertebral axis line on the posterior trunk view. Superior displacement $=A / H$. Downward/upward rotation around an axis perpendicular to the scapular plane is defined as the difference between the angles $\alpha$ defined by the vertebral axis and the extension of the lines connecting the center of the glenoid cavity and the medial end of the spine of the scapula. $\mathbf{c}$ In the anterior trunk view three lines are drawn: Line 1 is the line between the sternoclavicular joint and the center of the acromio-clavicular joint, line 2 is the line between the center of the acromio-clavicular joint and the inferior angle of the scapula, line 3 is the vertebral axis. The superior scapular angle $\gamma$ is the

angle defined between lines 1 and 2 and the inferior scapular angle $\delta$ is the angle between lines 2 and 3. d In the superior outlet view the anterior/posterior rotation is calculated as the angle $\phi$ between the line connecting the acromio-clavicular joint to the superomedial angle and the line between the sterno-clavicular joint and the center of the acromio-clavicular joint (clavicular axis). e Schematic drawing showing the method of calculating glenoscapular angle (glenoid version $\theta$ ), posterior subluxation of the humeral head and spinoscapular angle $(\zeta)$. The scapular line that connects the medial aspect of the scapula and the mid glenoid is drawn. A second line is drawn connecting the posterior and anterior margins of the glenoid. $90^{\circ}$ are subtracted from the angle of the posterior medial quadrant defined by these lines to determine the glenoid version $\theta$. A line perpendicular to the scapular line is drawn and the percentage of posterior subluxation is defined as the ratio of the distance from the scapular line to the anterior portion of the head to the diameter of the humeral head (LM/ $\mathrm{LN} \times 100)$. The spinoscapular angle $(\zeta)$ is measured as the angle between the scapular line and the medial plane 
clavicle and the line connecting the superomedial angle of the scapula to the acromio-clavicular joint (Fig. 2d).

The total area of the scapula was calculated on the scapular posterior view. The area of the scapula visible on the anterior view above the clavicle with reference to the contralateral side was measured on the trunk anterior view. For distance and area measurements, graphic software (Universal Desktop Ruler, AVPSoft.com) was used.

On a transverse CT image at the mid-glenoid level a scapular line was constructed that connected the medial margin of the scapula to the middle of the glenoid fossa. The glenoscapular angle (glenoid version) was measured according to Friedman et al. [5] as the angle between the scapular line (connecting the scapular medial margin to the middle of the glenoid) and the line connecting the base of the anterior labrum and posterior labrum. By definition $90^{\circ}$ are subtracted from the posteromedial quadrant angle to define the glenoscapular angle $(\theta)$ (Fig. 2e). The same scapular line is used to determine the degree of humeral head subluxation. The greatest diameter of the head was measured (LN) as the distance of the scapular line to the anterior portion of the head (LM). The percentage of subluxation was calculated as the ratio of these distances multiplied by 100 (Fig. 2e). Rotation about an approximately vertical axis (internal/external) was assessed by measuring the spinoscapular angle $(\zeta)$ between the scapular line and the median plane.

Both the affected and contralateral sides were assessed and the values discussed take into account comparison between sides unless specifically indicated.

\section{Statistics}

Descriptive statistics were calculated for each variable, including mean, standard deviation and range. Comparisons of means for continuous variables were performed by the Pearson product correlation coefficients and the paired Student $t$ test with Microsoft Excel 2003 software (Microsoft, Redmond, WA, USA). $P$ values were two-tailed, and $P$ values of $<0.05$ were considered to be significant.

\section{Results}

The patients were noted to have unilateral scapular elevation as a result of downward rotation to various extents. The 3D-CT data for the patients as well as the significance value between affected and contralateral side and the correlation coefficient of each variable to the area of affected scapula visible above the clavicle are summarized in Table 1. The area of scapula visible above the clavicle significantly correlated with downward rotational deformity $(r=-0.65)$, affected to contralateral height ratio $(r=-0.55)$, ratio of affected acromion to total width $(r=65)$, ratio of affected acromion to height ( $r=0.85$ ), affected to contralateral acromion length $(r=0.56)$, affected to contralateral area $(r=-0.57)$, superior scapular angle $(r=-0.84)$, inferior scapular angle $(r=0.83)$ and subluxation $(r=-0.80)$.

Age did not significantly correlate with any of the parameters measured.

Vertical displacement was noted to be either superior (15 patients) or inferior (15 patients) and did not significantly correlate with any of the parameters measured.

Downward rotational deformity was found to correlate with superior $(r=0.53)$ and inferior $(r=-0.53)$ scapular angle.

Internal/external rotation did not significantly correlate with any of the parameters measured although only three patients had external rotation and two no rotation.

Anterior/posterior rotation did not correlate significantly with any of the parameters measured although only one patient had posterior rotation and one no rotation.

Area of scapula was found to correlate with superior scapular angle $(r=0.60)$.

Affected acromion to height ratio correlated with affected superior scapular angle $(r=-0.81)$, inferior scapular angle $(r=0.83)$ and with subluxation $(r=-0.80)$. Affected to contralateral acromion to height ratio correlated with superior scapular angle $(r=-0.64)$, inferior scapular angle $(r=0.62)$ and with subluxation $(r=-0.62)$.

Superior and inferior scapular angles correlated with subluxation ( $r=0.63$ and $r=-0.63$, respectively).

Subluxation correlated with glenoscapular angle $(r=0.70)$.

A grading scale was devised for assessing scapular deformity in brachial plexus palsy, based on the 3D-CT of the observed patients. The different stages are illustrated in Fig. 3.

\section{Discussion}

The position of the affected scapula did not follow the symptoms and characteristics of Sprengel's deformity with congenital origin of scapular elevation. The hypoplasia and positioning of the scapula result from the 


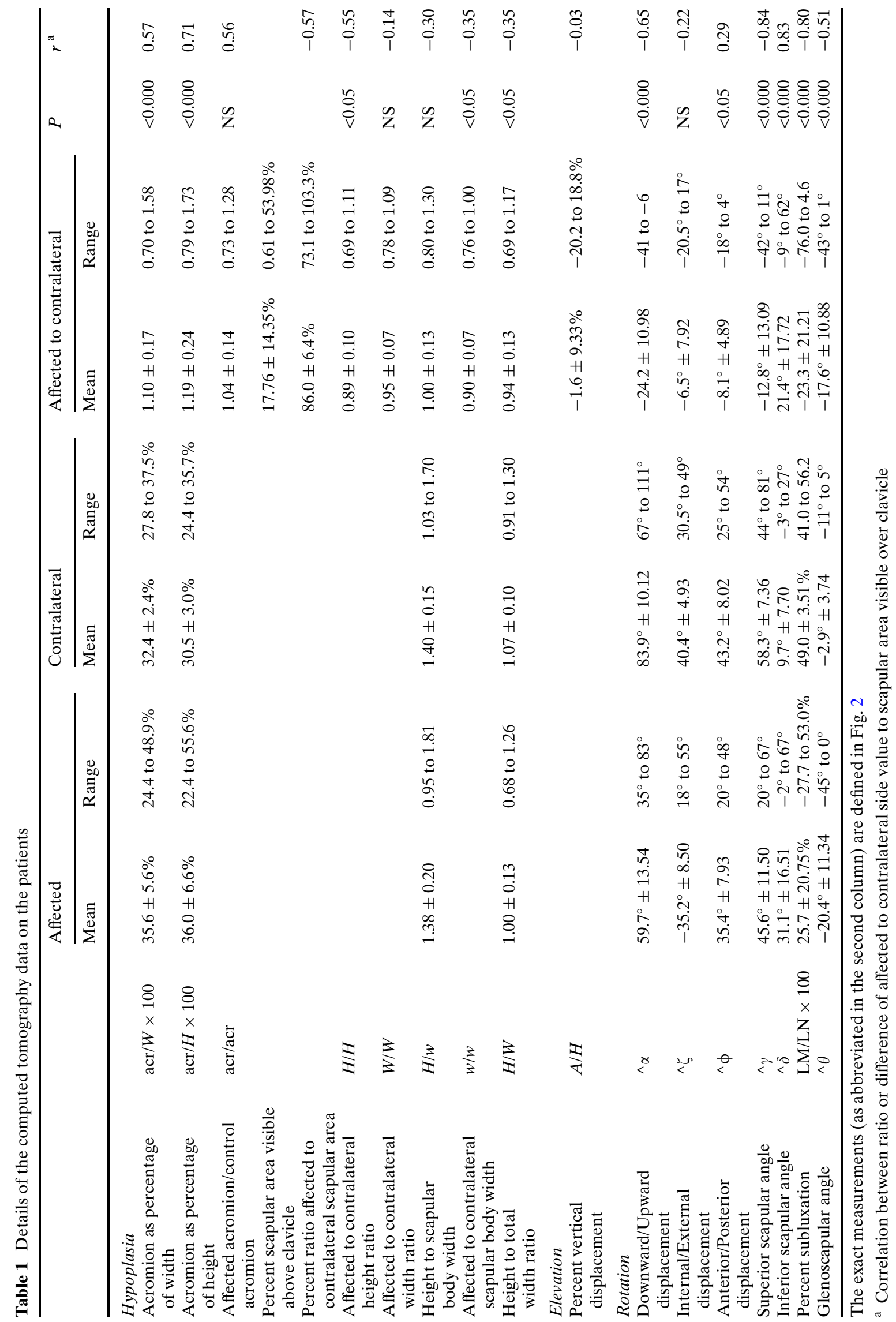



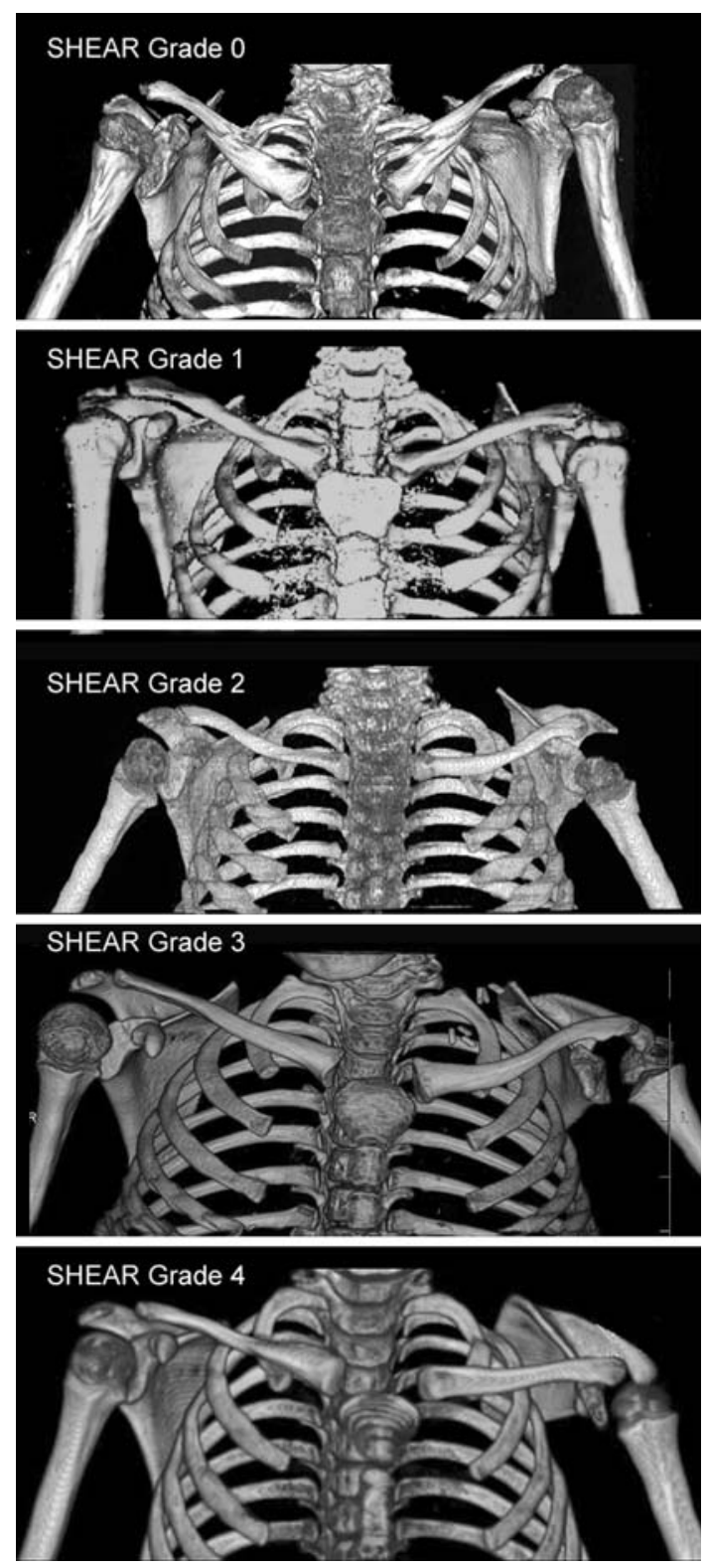

Fig. 3 The different SHEAR stages of scapular deformity as determined by three dimensional computer tomography. In SHEAR Grade 0 less than $2 \%$ of the scapula, less than $20 \%$ of the superior border and less than $6.5 \%$ of the medial border are visible above the clavicle. In SHEAR Grade $12-3.6 \%$ of the scapular area, $20-45 \%$ of the superior border and $6.5-16.5 \%$ of the medial border are visible over the clavicle. In SHEAR Grade $23.6-20 \%$ of the scapular area, $45-58 \%$ of the superior border and $16.5-$ $28 \%$ of the medial border are visible above the clavicle. In SHEAR Grade $320-45 \%$ of the scapula, $58-68 \%$ of the superior border and $28-50 \%$ of the medial border are visible over the scapula. In SHEAR Grade 4 more than $42 \%$ of the area of the scapula, more than $68.5 \%$ of the superior border and more than $50 \%$ of the medial border are visible over the scapula

brachial plexus injury and the apparent elevation is the result of downward rotation about an axis perpendicular to the scapular plane.
The lack of substantial forces applied to the scapula by the weakened scapula stabilizer muscles leads to a decreased rate of bone growth $[8,11]$. The observed decreased height to total width is partly the result of enlargement of the acromion, which may be caused by traction through the deltoid [7]. The acromion was found to be always tapered in the presence of the mildest deformity and often beaked and its ratio to both the total scapular width and height were significantly larger than the contralateral side (both $P<0.000$ ). The ratio of affected acromion to height correlated with the area of scapula visible over the clavicle $(r=0.85)$, with both affected superior scapular angle $(r=-0.81)$ and inferior scapular angle $(r=0.83)$ and with subluxation $(r=-0.80)$.

It seems that while the growth of height and width of the body of the scapula are impaired, the acromion follows a different pattern, of growth at the normal rate or at an aberrant rate if the deformity is more severe (correlation coefficient of affected to contralateral acromion and area of scapula visible over clavicle $r=0.56$ ) and often impinges on the humeral head.

The vertical displacement which was either negative or positive did not significantly correlate with any of the parameters assessed implying that the apparent elevation of the scapula is the result of rotational displacement unlike congenital scapular elevation where the severity of the deformity correlates with the level of the shoulder joint [4].

The affected scapula is not only malpositioned, it is also hypoplastic. Previous radiological assessments have used oblique images of the scapula $[8,11]$ and no report has utilized 3D-CT and measured these characteristics. In fact, in posterior trunk views the hypoplasia looks more exaggerated than by measurement in the scapular posterior view because the view is affected by the internal rotation. The total area of the hypoplastic scapula averages $14 \%$ less than the contralateral scapula and correlates with the severity of the deformity $(r=-0.57)$. While the rotation about a vertical axis was usually internal, in three instances it was external with no correlation to the severity of the deformity or to any other parameter assessed. Rotation about the axis of the scapular spine was in all but one instance anterior, forming a narrower scapuloclavicular space on the superior trunk outlet view.

During the development of scapular dysplasia, both downward rotational deformity (angle of the spine of the scapula) and decreased height to width ratio contribute to change in superior scapular angle and inferior scapular angle and both indeed strongly correlated with area of scapula visible above clavicle $(r=-0.75$ and $r=0.83)$ and degree of subluxation $(r=0.63$ and $r=-0.63)$. 
The clinical prognostic relevance of the various measurement methods is still under discussion $[1,13]$. Global abduction has been correlated with glenoid version [17]. In the literature there seems to be no significant correlation between Mallet functional parameters other than global abduction and either radiographic parameters or age $[13,17]$ because active shoulder function is affected by the glenohumeral relationship, shoulder capsule, strength and physical properties of the muscles and the nervous system.

In many patients, different versions of muscle release of the contracted internal rotators with or without muscle transfer of latissimus dorsi and teres major to the teres minor is performed early in life so that the weakened deltoid and supraspinatus muscles can achieve active abduction. The success of this intervention is very impressive and the abduction is often significantly improved $([6,10,15]$ and our unpublished data). While glenohumeral incongruence is delayed after muscle contracture release ([15] and our unpublished data), internal rotation of the arm and winging scapula often develop. The lack of correlation between age and any of the parameters of the anatomical deformity in our study, unlike reports by others [17], may reflect the fact that most of our patients had undergone contracture releases of the latissimus dorsi, teres major, subscapularis, and pectoralis major and minor with tendon transfer of latissimus dorsi and teres major. While global abduction in our series was near normal, the forces exerted by the muscles on the two scapulas are not equivalent obviously upholding impaired growth to the affected scapula. The rate of progression of the deformity is individual to the child.

The rotation of the scapula causes positional changes in the acromio-clavicular joint. This causes impingement of the distal acromion and clavicle with the humeral head, resulting in the MRC positioning described above. The clinical implications of the SHEAR and resulting MRC are clear and have been described by Birch in detail [2]. The current standard of practice for treatment of MRC is muscle transfer if the shoulder is congruent $[2,16]$, corresponding to SHEAR 0-1 and humeral derotational osteotomy if the humeral head is incongruent corresponding to SHEAR 2-4 [16]. While derotational osteotomy may restore external rotation by placing the arm in a more functional position it addresses neither the scapular nor the glenohumeral deformities. Our findings that SHEAR score based on the percentage of scapula visible over the clavicle strongly correlates with rotation of the scapula, hypoplasia and subluxation, provides a convenient diagnostic tool to assess the presence of the
MRC deformity. With the pathophysiology being thus established, anatomical correction of the deformity can be considered and planned, hopefully with improvement in functional parameters.

\section{Conclusion}

Scapular deformities common to the population of obstetric brachial plexus palsy patients due to muscular imbalances resulting from residual neurological deficit can be diagnosed and classified using the SHEAR classification and enable objective evaluation of the bony deformity and its severity as guide for treatment.

Acknowledgement The authors gratefully acknowledge the assistance of Sonya E. Melcher with statistical analysis and revision of the manuscript.

\section{References}

1. Al-Qattan MM (2003) Classification of secondary shoulder deformities in obstetric brachial plexus palsy. J Hand Surg [Br] 28(5):483-486. DOI 10.1016/S0266-7681(02)00399-6

2. Birch R (2003) Late sequelae at the shoulder in obstetric palsy in children. In: Duparc J (eds) Shoulder. Elsevier, Paris, pp 55-200-E-210

3. Birch R, Bonney G, Wynn Parry CB (1998) Birth lesions of the brachial plexus. In: Birch R, Bonney G, Wynn Parry CB (eds) Surgical disorders of the peripheral nerves. Shoulder, vol 3. Churchill Livingstone, New York, pp 209-233

4. Cho TJ, Choi IH, Chung CY, Hwang JK (2000) The Sprengel deformity. Morphometric analysis using 3D-CT and its clinical relevance. J Bone Joint Surg Br 82(5):711-718

5. Friedman RJ, Hawthorne KB, Genez BM (1992) The use of computerized tomography in the measurement of glenoid version. J Bone Joint Surg Am 74(7):1032-1037

6. Hoffer MM, Phipps GJ (1998) Closed reduction and tendon transfer for treatment of dislocation of the glenohumeral joint secondary to brachial plexus birth palsy. J Bone Joint Surg Am 80(7):997-1001

7. Kambhampati SB, Birch R, Cobiella C, Chen L (2006) Posterior subluxation and dislocation of the shoulder in obstetric brachial plexus palsy. J Bone Joint Surg Br 88(2):213-219. DOI 10.1302/0301-620X.88B2.17185

8. Kattan KR, Spitz HB (1968) Roentgen findings in obstetrical injuries to the brachial plexus. Radiology 91:462-467

9. Kozin SH (2004) Correlation between external rotation of the glenohumeral joint and deformity after brachial plexus birth palsy. J Pediatr Orthop 24(2):189-193

10. Pagnotta A, Haerle M, Gilbert A (2004) Long-term results on abduction and external rotation of the shoulder after latissimus dorsi transfer for sequelae of obstetric palsy. Clin Orthop Relat Res 426:199-205. DOI 10.1097/01.blo.0000138957.11939.70

11. Pollock AN, Reed MH (1989) Shoulder deformities from obstetrical brachial plexus paralysis. Skeletal Radiol 18(4):295-297

12. Terzis JK, Vekris MD, Okajima S, Soucacos PN (2003) Shoulder deformities in obstetric brachial plexus paralysis: a computed tomography study. J Pediatr Orthop 23(2):254-260 
13. van der Sluijs JA, van der Meij M, Verbeke J, Manoliu RA, Wuisman PI (2003) Measuring secondary deformities of the shoulder in children with obstetric brachial plexus lesion: reliability of three methods. J Pediatr Orthop B 12(3):211-214

14. van der Sluijs JA, van Ouwerkerk WJ, de Gast A, Wuisman PI, Nollet F, Manoliu RA (2001) Deformities of the shoulder in infants younger than 12 months with an obstetric lesion of the brachial plexus. J Bone Joint Surg Br 83(4):551-555

15. Waters PM, Bae DS (2005) Effect of tendon transfers and extra-articular soft-tissue balancing on glenohumeral develop- ment in brachial plexus birth palsy. J Bone Joint Surg Am 87(2):320-325. DOI 10.2106/JBJS.C.01614

16. Waters PM, Bae DS (2006) The effect of derotational humeral osteotomy on global shoulder function in brachial plexus birth palsy. J Bone Joint Surg Am 88(5):1035-1042. DOI 10.2106/JBJS.E.00680

17. Waters PM, Smith GR, Jaramillo D (1998) Glenohumeral deformity secondary to brachial plexus birth palsy. J Bone Joint Surg Am 80(5):668-677 